\title{
Sport and physical activity after ankle arthrodesis with llizarov fixation and internal fixation
}

\author{
Piotr Morasiewicz, ${ }^{1, A-F}$, Maciej Dejnek ${ }^{1, A-F}$, Mirosław Kulej ${ }^{1, A, D-F}$, Szymon . Dragan $^{1, A, D-F}$, Grzegorz Konieczny, ${ }^{2, A, D-F}$, \\ Artur Krawczyk ${ }^{1, A, D-F}$, Wiktor Urbański ${ }^{1, A, D-F}$, Wiktor Orzechowski ${ }^{1, A, D-F}$, Szymon F. Dragan ${ }^{1, A, D-F}$, Łukasz Pawik ${ }^{3, A, D-F}$ \\ ${ }^{1}$ Department and Clinic of Orthopaedic and Traumatologic Surgery, Faculty of Medicine, Wroclaw Medical University, Poland \\ ${ }^{2}$ Faculty of Health Sciences and Physical Education, Witelon State University of Applied Sciences in Legnica, Poland \\ ${ }^{3}$ Department of Physiotherapy and Occupational Therapy in Motor Disorders and Dysfunctions, University School of Physical Education in Wroclaw, Poland \\ A - research concept and design; $\mathrm{B}$ - collection and/or assembly of data; $\mathrm{C}$ - data analysis and interpretation; \\ $D$ - writing the article; $E$ - critical revision of the article; $F$ - final approval of the article
}

\section{Address for correspondence \\ Piotr Morasiewicz}

E-mail:morasp@poczta.onet.pl

\section{Funding sources}

Internal university grant: ST.C080.16.073.

Conflict of interest

None declared

Received on May 15, 2017

Reviewed on June 26, 2017

Accepted on November 14, 2017

Published online on August 6, 2018

Cite as

Morasiewicz P, Dejnek M, Kulej M, et al. Sport and physical activity after ankle arthrodesis with llizarov fixation and internal fixation. Adv Clin Exp Med. 2019;28(5):609-614. doi:10.17219/acem/80258

DOI

10.17219/acem/80258

\section{Copyright}

Copyright by Author(s)

This is an article distributed under the terms of the Creative Commons Attribution Non-Commercial License (http://creativecommons.org/licenses/by-nc-nd/4.0/)

\begin{abstract}
Background. Severe osteoarthritis $(\mathrm{OA})$ of the ankle joint constitutes an important social problem.

Objectives. We used (1) the GRIMBY scale, (2) the LOWER LIMB Activity scale, (3) the UCLA (University of California Los Angeles) activity scale, (4) the VAS (visual analogue scale) ACTIVITY scale, and (5) the FAAM (foot and ankle ability measure) SPORT scale to verify whether the type of ankle joint arthrodesis stabilization affected sports and physical activity levels.
\end{abstract}

Material and methods. We carried out a prospective clinical study of 47 patients who had undergone ankle arthrodesis with llizarov external fixator stabilization (Group 1, $n=21$ ) or internal stabilization with screws (Group 2, n= 26) at Orthopaedic Clinic at the Wroclaw Medical University, Poland, from 2007 to 2015. Sports and physical activity levels were measured by (1) the GRIMBY scale, (2) the LOWER LIMB Activity scale, (3) the UCLA activity scale, (4) the VAS ACTIVITY scale, and (5) the FAAM SPORT scale.

Results. A comparison between the average results of Group 1 and Group 2 on the LOWER LIMB Activity scale and the GRIMBY scale before and after surgery revealed no significant differences. In Group 1, the mean scores on the VAS ACTIVITY scale and the UCLA activity scale after treatment were higher than in Group 2. In Group 1, the mean outcome in the SPORT FAAM scale after treatment was 40; in Group 2 it was 30.06.

Conclusions. llizarov fixation of ankle arthrodesis is associated with better scores on the FAAM SPORT, UCLA activity and VAS ACTIVITY scales after treatment than internal fixation. The scores on the GRIMBY scale and the UCLA activity scale were significantly higher after treatment than before treatment in both groups. In this study, ankle fusion with lizarov fixation and internal fixation was found to be effective in the treatment of ankle arthritis. The levels of sport and physical activity were satisfactory in both groups, but the outcomes after fixation with the llizarov apparatus were better than after internal stabilization.

Key words: sport, physical activity, internal fixation, ankle arthrodesis, lizarov fixation 


\section{Introduction}

Severe osteoarthritis (OA) of the ankle joint constitutes an important social problem. It results in functional disturbances and in compensation of irregularities, which are more pronounced while moving and standing. ${ }^{1-5}$ This leads to poorer functioning of the lower limb, lower muscle strength, deterioration in gait efficiency, and reduced mobility of joints, which reduces the physical activity and participation in sports. ${ }^{1-5}$ Ankle arthrodesis has been the gold standard operative treatment for end-stage ankle arthritis. ${ }^{4,6-19}$ Ankle arthrodesis can be achieved by external fixation or internal fixation. ${ }^{6-8,11-13,16-20}$ An operation is effective when it reduces pain and improves the function of the limb. ${ }^{1,2,11,20,21}$ Nowadays, patients tend to quickly return to work, independence and sports activities. ${ }^{3,4}$ Ankle arthrodesis may be associated with a reduction in physical activity and sport after surgery. ${ }^{2-5}$

An optimal method for ankle arthrodesis should provide a good functional outcome and should enable the patient to be involved in sports and other forms of physical activity to the same or even a greater extent than prior to the procedure. $^{1-5}$

All of the studies report sports and physical activity levels separately for each of the 2 ankle arthrodesis procedures: Ilizarov fixation and internal fixation. ${ }^{2,4,5}$ There is no study in the literature comparing sport and physical activity level with the GRIMBY scale, the LOWER LIMB Activity scale, the UCLA (University of California Los Angeles) activity scale, the VAS (visual analogue scale) ACTIVITY scale, and the FAAM (foot and ankle ability measure) SPORT scale in both groups with Ilizarov fixation and internal fixation of ankle arthrodesis.

We hypothesized that the type of ankle arthrodesis stabilization does not affect sports and physical activity levels. Our objective was to verify whether the type of ankle joint arthrodesis stabilization affected sports and physical activity levels.

\section{Material and methods}

We carried out a retrospective clinical study of all 55 patients who had undergone ankle arthrodesis with Ilizarov external fixator stabilization (Group 1) or internal stabilization with screws (Group 2) at Orthopaedic Clinic at the Wroclaw Medical University, Poland, from 2007 to 2015. The indications of ankle arthrodesis included severe primary or secondary (post-traumatic, neurogenic, rheumatoid, or congenital) degenerative/deforming changes of the ankle joint. $^{22}$

Patients were subjected to ankle arthrodesis with either external Ilizarov fixation or internal fixation with cannulated screws. Individuals with a poor status of the soft tissues (necrosis, inflammation, fistulas, trophic changes, scars, vascular lesions, skin lesions, or ulcers) or bones (osteoporotic, bone loss or infections), severe deformities
( $>15^{\circ}$ in 1 axis or multiplanar deformities), or infection were always qualified for ankle arthrodesis with Ilizarov fixation. The remaining patients were subjected to ankle arthrodesis with either external Ilizarov fixation or internal fixation with cannulated screws. We preferred ankle arthrodesis with internal fixation in patients with a good status of the soft tissues and bones, deformities $<5^{\circ}$ and without any concomitant disease which prevented immobilization in a plaster cast, and in patients who cooperated with restricted limb weight bearing just after the surgery. ${ }^{22}$

The criteria for inclusion in the study consisted of: performance of ankle arthrodesis with Ilizarov external fixator stabilization or internal stabilization with cannulated screws; more than 24 months from the conclusion of the treatment; the presence of baseline values of ankle pathology etiology and demographic data in medical records; and the presence of preoperative and postoperative sport and physical activity level scores (the GRIMBY scale, the LOWER LIMB Activity scale, the UCLA activity scale, the VAS ACTIVITY scale, and the FAAM SPORT scale).

The exclusion criteria consisted of: ankle arthrodesis without Ilizarov external fixator stabilization or internal stabilization with cannulated screws; a lack of baseline values of ankle pathology etiology and/or demographic data in medical records; a lack of preoperative and postoperative sport and physical activity level scores; a follow-up period shorter than 24 months; Charcot neuroarthropathy; multiple joint or bilateral ankle injuries; and undergoing associated procedures during surgical intervention.

Patients were included in the study based on medical history, medical record analysis performed before and after treatment, and physical examination. The participants were informed about the voluntary nature of the study and they gave their consent to participate in the study. In the case of minors, consent was given by their legal guardians. The study was approved by the Wroclaw Medical University Bioethics Commission.

Between 2007 and 2015, 55 patients underwent ankle arthrodesis. Of these, 24 were treated with an Ilizarov device and 31 with internal fixation (screws). In the Ilizarov group, 1 patient (4\%) was lost to follow-up during the 2 years, 1 (4\%) was not included because of missing data in the patient's records and 1 (4\%) was excluded because of neuropathic arthropathy, bilateral ankle injuries or associated ankle procedures at the time of the arthrodesis, leaving a total of 21 patients for analysis. In the internal fixation group, 2 patients were lost to follow-up during the 24 months (6\%), 2 (6\%) were not included because of missing data in their records and 1 (3\%) was excluded because of neuropathic arthropathy, bilateral ankle injuries or associated ankle procedures at the time of the arthrodesis, leaving a total of 26 patients for analysis.

All patients were given perioperative antibiotics and were placed in a supine position; then a tourniquet was applied (320 $\mathrm{mm} \mathrm{Hg})$. An anterior approach centered over the ankle joint was used for ankle joint fusion. An Ilizarov 
apparatus (Group 1) or cannulated screws (Group 2) were used to create compression at the ankle joint. The Ilizarov apparatus for ankle arthrodesis consisted of a proximal ring fixed to the tibia and fibula with 3 Kirschner wires, a distal ring fixed to the tibia and fibula with 2 Kirschner wires, and a U-shaped foot ring fixed to the calcaneus with 2 Kirschner olive wires and fixed to the distal part of the metatarsal bones with 1 Kirschner olive wire. All patients in Groups 1 and 2 were operated on by 3 surgeons. Patients from Group 1 (Ilizarov stabilization) started weight bearing on postoperative day 1 . The minimum time of wearing the Ilizarov fixator was 9 weeks. After the Ilizarov fixator was removed, patients transitioned to a walker boot for a minimum of 6 weeks. Postoperatively, patients from Group 2 remained non-weight bearing for a minimum of 6 weeks in a cast, followed by protected progressive weight bearing in a controlled ankle motion walker (CAM walker) for the next 6 weeks. Usually, by 3 months patients had made a transition to wearing normal shoes. ${ }^{22}$

The patients' sports and physical activity levels were assessed using the GRIMBY scale, the LOWER LIMB Activity scale, the UCLA activity scale, the VAS ACTIVITY scale, and the FAAM SPORT scale. ${ }^{20,23-26}$ Mean preoperative and postoperative scores have been calculated.

To verify whether the average value of the variables varied significantly, the Mann-Whitney U test and Student's t-test were used. To analyze the significance of differences between the mean values of data variables in Groups 1 and 2, we used the Mann-Whitney U test and Student's t-test. Where the use of test data required fulfillment of assumptions about normality, the Shapiro-Wilk test and Kolmogorov-Smirnov test were used to verify the hypothesis of normality. All analyses were carried out at an assumed significance level of $\alpha=0.05$ using STATISTICA v. 10.0 software (StatSoft Inc., Tulsa, USA).

\section{Results}

There was no statistically significant difference in the demographic data for patients in Groups 1 and 2 (Table 1).

Frontal plane alignment of the ankle joint was observed in $100 \%$ of the patients from the Ilizarov group and in $75.8 \%$ from the internal fixation group. The sagittal plane alignment rates were $100 \%$ in Group 1 and $84.8 \%$ in Group 2. Ankle fusion was achieved in $100 \%$ of the patients in Group 1 and in $87.9 \%$ in Group 2.

In the group with Ilizarov fixation (Group 1), the average rate of complications was 0.62 complications per patient. In the group with internal stabilization, there were 0.58 complications per patient. In Group 1, 90.5\% of the patients were very satisfied or satisfied with the treatment. In the group with internal fixation with cannulated screws (Group 2), 88.5\% of the patients were very satisfied or satisfied with the treatment.

In Group 1, the median activity score on the GRIMBY scale before treatment was 3. The median GRIMBY scale

Table 1. Demographic characteristics of the patients

\begin{tabular}{|c|c|c|c|}
\hline Variable & $\begin{array}{l}\text { Group } 1 \text { - llizarov external } \\
\text { fixator }(n=21)\end{array}$ & $\begin{array}{l}\text { Group } 2 \text { - internal stabilization } \\
\qquad(\mathrm{n}=26)\end{array}$ & $p$-value \\
\hline Age [years] & $44(17-65)$ & $47(17-67)$ & $p=0.24$ \\
\hline Sex & $14(66.6 \%)$ male & $17(65.4 \%)$ male & $p=0.45$ \\
\hline Follow-up [months] & $45(24-108)$ & $47(24-104)$ & $p=0.38$ \\
\hline Disease diagnosis & - & - & - \\
\hline primary OA & $2(9.5 \%)$ & $3(11.5 \%)$ & $p=0.29$ \\
\hline secondary OA & - & - & - \\
\hline post-traumatic & $10(47.6 \%)$ & $15(57.7 \%)$ & $p=0.12$ \\
\hline rheumatoid & $0(0 \%)$ & $1(3.8 \%)$ & $p=0.13$ \\
\hline congenital & $4(19 \%)$ & $3(11.5 \%)$ & $p=0.16$ \\
\hline neuropathic & $5(23.8 \%)$ & $4(15.4 \%)$ & $p=0.21$ \\
\hline SPORT FAAM scale score after treatment (0-100) & median 41; mean $40(11-100)^{*}$ & median 30; mean $30.06(14-100)^{*}$ & $p=0.041$ \\
\hline GRIMBY scale score before treatment (1-6) & median 3; mean $3.23(1-6)$ & median 3; mean $3.12(1-6)$ & $p=0.29$ \\
\hline GRIMBY scale score after treatment (1-6) & median 4.5; mean 4.45 (1-6) & median 4.5; mean $4.42(1-6)$ & $p=0.49$ \\
\hline LOWER LIMB Activity scale score before treatment (1-18) & median 9.5; mean 9.63 (4-18) & median 9.5; mean 9.7 (2-15) & $p=0.51$ \\
\hline LOWER LIMB Activity scale score after treatment (1-18) & median 12; mean 12.25 (7-15) & median 12; mean 11.77 (3-17) & $p=0.19$ \\
\hline UCLA activity scale score before treatment (1-10) & median 5; mean 4.93 (2-10) & median 5; mean 4.98 (2-9) & $p=0.41$ \\
\hline UCLA activity scale score after treatment (1-10) & median 7; mean $6.97(3-9)^{*}$ & median 6; mean $5.97(2-9)^{*}$ & $p=0.033$ \\
\hline VAS ACTIVITY scale score before treatment (0-10) & median 5; mean $5.13(1-10)$ & median 5; mean 5 (0-10) & $p=0.32$ \\
\hline VAS ACTIVITY scale score after treatment (0-10) & median 7; mean $6.85(2-10)^{*}$ & median 5.5; mean $5.35(2-10)^{*}$ & $p=0.043$ \\
\hline
\end{tabular}

* statistical difference between the groups ( $p<0.05)$; OA - osteoarthritis; UCLA - University of California Los Angeles; VAS - visual analogue scale; FAAM - foot and ankle ability measure. 
score after treatment increased to 4.5 and these values were statistically different $(\mathrm{p}=0.041)$. In the group with internal stabilization, the median value of GRIMBY activity level before treatment was 3. The median GRIMBY scale score after treatment was 4.5 . These values were statistically different $(\mathrm{p}=0.045)$ (Table 1$)$.

In Group 1, the median value of the LOWER LIMB Activity scale score before treatment was 9.5. The median LOWER LIMB Activity scale score after treatment was 12. These values were not statistically different $(p=0.13)$. In the group with internal stabilization, the median score on the LOWER LIMB Activity scale before treatment was 9.5. The median LOWER LIMB Activity scale score after treatment was 12 (Table 1). These values were not statistically different $(\mathrm{p}=0.16)$.

In Group 1, the median score on the UCLA activity scale before treatment was 5 . The median UCLA activity scale score after treatment was 7 . These values were statistically different $(\mathrm{p}=0.041)$. In the group with internal stabilization, the median score on the UCLA activity scale before treatment was 5 . The median UCLA activity scale score after treatment was 6 (Table 1). These values were statistically different $(\mathrm{p}=0.047)$. The UCLA activity scale values after treatment for Group 1 were significant higher than for Group 2 ( $\mathrm{p}=0.033)$.

In Group 1, the median score on the VAS ACTIVITY scale before treatment was 5 . The median VAS ACTIVITY scale score after treatment was 7 . These values were statistically different ( $\mathrm{p}=0.039$ ). In the group with internal stabilization, the median score on the VAS ACTIVITY scale before treatment was 5 . The median VAS ACTIVITY scale score after treatment increased to 5.5 (Table 1). These values were not statistically different $(\mathrm{p}=0.34)$. After treatment, the group with Ilizarov stabilization (Group 1) had significantly higher VAS ACTIVITY scale scores (Table 1$)(p=0.043)$.

The FAAM functional outcome was significantly higher in the group with Ilizarov stabilization (Table 1) $(\mathrm{p}=0.041)$.

\section{Discussion}

The method for ankle joint arthrodesis should allow for the practice of sports and physical activity after treatment at the highest possible level.

To the best of our knowledge, none of the previous studies analyzed sports and physical activity levels after ankle arthrodesis regarding the fixation method used. MacMahon et al. analyzed sports and physical activity levels in 38 patients who had undergone primary partial arthrodesis for a Lisfranc injury. ${ }^{4}$ Preoperatively, $47.1 \%$ engaged in high-impact sports, and postoperatively, $44.8 \%$ did. Compared to the preoperative levels, the difficulty was the same in $66 \%$ and had increased in $34 \%$ of physical activities. Participation levels improved in $11 \%$, remained the same in $64 \%$ and were impaired in $25 \%$ of physical activities. The decrease in participation or increase in difficulty of some activities suggests that some patients experienced postoperative limitations in exercise. ${ }^{4}$

As far as we know, none of the previous studies compared GRIMBY scale scores in patients subjected to ankle arthrodesis with external and internal fixation. Morasiewicz et al. analyzed sports and physical activity levels in 56 patients after derotational osteotomy with the Ilizarov method; the mean level of activity on the GRIMBY scale after treatment was $4.2 .^{3}$ Their findings are similar to those obtained in our present study. The mean GRIMBY scale scores after treatment increased statistically significantly in both groups.

None of the previous studies compared the values of the LOWER LIMB Activity scale score in patients after ankle arthrodesis with external and internal fixation. The mean value of the LOWER LIMB Activity scale in patients examined by Morasiewicz et al. was $11.84 .^{3}$ Their findings are similar to those obtained in the present study. In our study, patients from both groups presented with similar pre- and post-treatment values of the LOWER LIMB Activity scale score. The post-treatment values of the LOWER LIMB Activity scale score are higher than pretreatment values, but not statistically significantly.

None of the previous studies compared the values of the UCLA activity scale score in patients with external and internal fixation of ankle arthrodesis. Schuh et al. used this scale to examine 21 patients after ankle arthrodesis; the mean postoperative UCLA activity scale score in this group was 7.0. ${ }^{5}$ According to Morasiewicz et al., the mean post-treatment UCLA activity scale score in 56 patients subjected to derotational osteotomy with the Ilizarov method was $6.18 .^{3}$ Their findings are similar to those obtained in our present study. Irrespective of fixation type, we did not observe statistically significant differences between preoperative UCLA activity scale scores. The posttreatment values of the UCLA activity scale score are higher than the pretreatment values in both groups. The average score on the UCLA activity scale after treatment was significant higher for the Ilizarov group than for the internal fixation group.

To the best of our knowledge, none of the previous studies compared the values of the VAS ACTIVITY scale in individuals with ankle arthrodesis with external and internal fixation. In the study conducted by Morasiewicz et al., mean VAS ACTIVITY scale score after treatment was 5.98, i.e., similar to our present study. ${ }^{3}$ We observed a significant postoperative increase in the VAS ACTIVITY scale scores of patients with Ilizarov fixation; the post-treatment scores in this group turned out to be significantly better than in subjects with internal fixation of ankle arthrodesis.

As far as we know, the results of the FAAM SPORT scale in patients with external and internal fixation of ankle arthrodesis have not been thus far subjected to a comparative analysis. According to Dalat et al., the mean postoperative FAAM SPORT scale score for a group of 46 patients after ankle arthrodesis with internal fixation was 29.8 ; this value 
was slightly lower than the one we found in our subjects. ${ }^{2}$ In our present study, individuals with Ilizarov fixation presented with better FAAM SPORT scale scores than those subjected to internal fixation.

Patients with Ilizarov fixation had better FAAM SPORT scale scores, UCLA activity scale scores and VAS ACTIVITY scale scores after treatment than those after internal fixation. Ilzarov fixation exerts lesser impact on musculoskeletal biomechanics than in the case of internal fixation. Also, the possibility of fully loading the treated limb soon after fixation with the Ilizarov apparatus seems to be more beneficial than completely sparing the extremity after internal stabilization. The better sports and physical activity results of arthrodesis with external fixation can be related to better frontal and sagittal plane alignment of the ankle joint and higher ankle fusion rate. However, irrespective of the study group, the sports and physical activity level scores of our patients were close to those reported by other researchers. ${ }^{2,5}$ According to some authors, the sports and physical activity level outcomes of ankle arthrodesis are mediocre. ${ }^{2,4,5}$ In a study conducted by Chahal et al., the mean functional scores of patients subjected to ankle arthrodesis with internal fixation were below the reference values for the American population. ${ }^{1}$ In a study conducted by Dalat et al., the overall mean athletic level after the surgery was relatively low compared to the state prior to the injury. ${ }^{2}$ After ankle arthrodesis, the number of patients participating in sports decreased. ${ }^{5}$ McKee et al. noticed that $64 \%$ of patients with post-traumatic deformity correction with the Ilizarov method could return to physical activity, but at a reduced level; this study did not provide a detailed description of the types of activity. ${ }^{3,27}$

In general, patients who had ankle joint arthrodesis can expect worse sports and physical activity levels than the general population. ${ }^{4}$ This would indicate that they experience pain and a higher level of disability than the general population. Improper function of the lower limbs limits and even prevents participation in sports and physical activity. Worse limb function may result from instability and limitation of movement of the joints, from pain, reduced muscle strength, connective tissue scars, and increased body weight. ${ }^{3}$ We noticed an improvement in the sports and physical activity levels of patients from both groups. The lack of a very significant improvement in sports and physical activity levels might reflect a post-arthrodesis disruption of lower extremity biomechanics. Significant asymmetry in gait and a reduced range of motion remained after ankle joint arthrodesis compared to normal. ${ }^{28}$

One potential limitation of our present study may stem from the fact that the FAAM SPORT scale scores were determined solely postoperatively. However, the values of all other activity scales were determined both before and after treatment. The patients in both groups were operated on by 3 different surgeons, but following the same protocol. The strong points of this study are the homogeneity of the techniques and surgical recovery in both groups.
Importantly, none of the previous studies compared the sports and physical activity levels after ankle arthrodesis with 5 different activity scales.

This study was not randomized. Both groups were similar in age, etiology, gender, and follow-up time. Ilizarov arthrodesis was predominant in patients with a poor status of soft tissues and bones, severe deformities and infection. These factors, which we used as criteria for Ilizarov external fixator stabilization, could have impacted the final results. Theoretically, patients in Group 1 had more negative influences on sports and physical activity levels before surgery. Thus, postoperative sports and physical activity levels should have improved more in Group 1 than in Group 2. Ankle arthrodesis with internal fixation was performed in patients with a good status of soft tissues and bones, with deformities $<5^{\circ}$, and without concomitant diseases or infection. Supposedly, patients in Group 2 had fewer adverse factors for sports and physical activity. Treatment with cannulated screws was less stressful. Theoretically, sports and physical activity levels after arthrodesis with cannulated screws may be less improved than Group 1.

In this paper, we compared only sports and physical activity after ankle arthrodesis with Ilizarov fixation and internal fixation; as in our other published works, we evaluated the radiological outcomes and clinical outcomes in patients after ankle arthrodesis with Ilizarov fixation and internal fixation. ${ }^{22}$

Ankle arthrodesis with Ilizarov fixation and internal stabilization resulted in normalization of lower limb loads. Balance after ankle arthrodesis with Ilizarov fixation is worse than with internal stabilization ankle arthrodesis.

Ilizarov fixation of ankle arthrodesis is associated with better FAAM SPORT scale scores, UCLA activity scale scores and VAS ACTIVITY scale scores after treatment than after internal fixation. The scores of the GRIMBY scale and the UCLA activity scale after treatment were significantly higher than before treatment in both groups.

In this study, ankle fusion with Ilizarov fixation and internal fixation was found to be effective in the treatment of ankle arthritis. Sports and physical activity levels were satisfactory in both groups, but the outcomes after fixation with the Ilizarov apparatus are better than after internal stabilization.

\section{References}

1. Chahal J, Stephen DJ, Bulmer B, Daniels T, Kreder HJ. Factors associated with outcome after subtalar arthrodesis. J Orthop Trauma. 2006;20(8):555-561.

2. Dalat F, Trouillet F, Fessy MH, Bourdin M, Besse JL. Comparison of quality of life following total ankle arthroplasty and ankle arthrodesis: Retrospective study of 54 cases. Orthop Traumatol Surg Res. 2014;100(7):761-766.

3. Morasiewicz P, Konieczny G, Pawik $Ł$, Dragan S. Sport and physical activity in patients after derotational corticotomies with the llizarov method. Acta Orthop Belg. 2015;81:90-99.

4. MacMahon A, Kim P, Levine D, et al. Return to sports and physical activities after primary partial arthrodesis for Lisfranc injuries in young patients. Foot Ankle Int. 2016;37(4):355-362. 
5. Schuh R, Hofstaetter J, Krismer M, Bevoni R, Windhager R, Trnka HJ. Total ankle arthroplasty versus ankle arthrodesis: Comparison of sports, recreational activities and functional outcome. Int Orthop. 2012;36(6):1207-1214

6. Fragomen AT, Borst E, Schachter L, Lyman S, Rozbruch RS. Complex ankle arthrodesis using the llizarov method yields high rate of fusion. Clin Orthop Relat Res. 2012;470(10):2864-2873.

7. Rabinovich RV, Haleem AM, Rozbruch SR. Complex ankle arthrodesis: Review of the literature. World J Orthop. 2015;6(8):602-613.

8. Siebachmeyer M, Boddu K, Bilal A, et al. Outcome of one-stage correction of deformities of the ankle and hindfoot and fusion in Charcot neuroarthropathy using a retrograde intramedullary hindfoot arthrodesis nail. Bone Joint J. 2015;97-B(1):76-82.

9. Perlman $\mathrm{MH}$, Thordarson DB. Ankle fusion in a high risk population: An assessment of nonunion risk factors. Foot Ankle Int. 1999;20(8): 491-496.

10. Jang JJ, Schipper ON, Whyte N, Koh JL, Toolan BC. Comparison of perioperative complications and hospitalization outcomes after ankle arthrodesis versus total ankle arthroplasty from 2002 to 2011. Foot Ankle Int. 2015;36(4):360-368.

11. Tellisi N, Fragomen AT, llizarov S, Rozbruch SR. Limb salvage reconstruction of the ankle with fusion and simultaneous tibial lengthening using the llizarov/Taylor spatial frame. HSS J. 2008;4(1):32-42.

12. Katsenis D, Bhave A, Paley D, Herzenberg JE. Treatment of malunion and nonunion at the site of an ankle fusion with the llizarov apparatus. J Bone Joint Surg Am. 2005;87(2):302-329.

13. Onodera T, Majima T, Kasahara $Y$, et al. Outcome of transfibular ankle arthrodesis with Ilizarov apparatus. Foot Ankle Int. 2012;33(11):964-968.

14. Salem KH, Kinzl L, Schmelz A. Ankle arthrodesis using llizarov ring fixators: A review of 22 cases. Foot Ankle Int. 2006;27(10):764-770.

15. Ling JS, Smyth NA, Fraser EJ, et al. Investigating the relationship between ankle arthrodesis and adjacent-joint arthritis in the hindfoot: A systematic review. J Bone Joint Surg Am. 2015;97(6):513-520.

16. Houdek MT, Wilke BK, Ryssman DB, Turner NS. Radiographic and functional outcomes following bilateral ankle fusions. Foot Ankle Int 2014;35(12):1250-1254.
17. Strasser NL, Turner NS. Functional outcomes after ankle arthrodesis in elderly patients. Foot Ankle Int. 2012;33(9):699-703.

18. Hendrickx RP, Stufkens SA, de Bruijn EE, Sierevelt IN, van Dijk CN, Kerkhoffs GM. Medium- to long-term outcome of ankle arthrodesis. Foot Ankle Int. 2011;32(10):940-947.

19. Slater GL, Sayres SC, O'Malley MJ. Anterior ankle arthrodesis. World J Orthop. 2014;5(1):1-5.

20. Martin RL, Irrgang JJ, Burdett RG, Conti SF, Van Swearingen JM. Evidence of validity for the Foot and Ankle Ability Measure (FAAM). Foot Ankle Int. 2005;26(11):968-983.

21. Bednarz $P$, Beals $T$, Manila A. Subtalar distraction bone blok fusion: An assessment of outcome. Foot Ankle Int. 1997;18(12):785-791.

22. Morasiewicz P, Dejnek M, Urbański W, Dragan SŁ, Kulej M, Dragan SF. Radiological evaluation of ankle arthrodesis with llizarov fixation compared to internal fixation. Injury. 2017;48(7):1678-1683.

23. Grimby G. Physical activity: Muscle training in the elderly. Acta Medica Scand Suppl. $1986 ; 711: 233-237$.

24. Saleh K, Mulhall K, Bershadsky B, et al. Development and validation of a lower-extremity activity scale: Use for patients treated with revision total knee arthroplasty. J Bone Joint Surg Am. 2005;87(9): 1985-1994.

25. Amstutz H, Thomas B, Jinnah R, Kim W, Grogan T, Yale C. Treatment of primary osteoarthritis of the hip: A comparison of total join and surface replacement arthroplasty. J Bone Joint Surg Am. 1984;66(2):228-241.

26. Zahiri C, Schmalzried T, Szuszczewicz E, Amstutz HC. Assessing activity in joint replacement patients. J Arthroplasty. 1998;13(8): 890-895.

27. McKee M, Yoo D, Schemitsch E. Health status after Ilizarov reconstruction of post-traumatic lower-limb deformity. J Bone Joint Surg Br. 1998;80(2):360-364.

28. Braito M, Dammerer D, Kaufmann G, et al. Are our expectations bigger than the results we achieve? A comparative study analyzing potential advantages of ankle arthroplasty over arthrodesis. Int Orthop. 2014;38(8):1647-1653. 